\title{
Real-time object control system using open source platform
}

\author{
Chang-Gyu Cgseong ${ }^{1}$, Jung-Yee Kim², Doo-Jin Park ${ }^{3}$ \\ ${ }^{1}$ Division of Software Education Center, Pusan National University, Korea \\ ${ }^{2,3}$ Department of Port Logistics System, Tongmyong University, Korea
}

\begin{tabular}{l}
\hline \hline Article Info \\
\hline Article history: \\
Received Jan 26, 2020 \\
Revised Mar 28, 2020 \\
Accepted Apr 14, 2020
\end{tabular}

Keywords:

IoT

MQTT protocol

Node-RED

Open source hardware

Real time object control

\begin{abstract}
Recently, the Internet of things(IoT) has received great attention, and the demand for IOT applications in various fields is increasing. But drawbacks of IoT, such as having to use dedicated equipment and having to pay for a flat fee monthly, do not satisfy the consumers' demands. These shortcomings of IoT is causing the appearance of users who try to design the environment of IoT that responds their demands and naturally, attempts to have monitoring system through open-source hardware like Arduino. Open source hardware has attracted a great deal of attention for the diffusion of the Internet of things as a key element of the Internet construction. The emergence of open source hardware, which has the advantage of low cost and easy and fast development, has made it possible to embody the idea of object Internet application services. In this paper, we design and implement a system that controls the objects in real time using open source hardware and MQTT protocol.
\end{abstract}

Copyright $\odot 2020$ Institute of Advanced Engineering and Science. All rights reserved.

\section{Corresponding Author:}

Jung-Yee, Kim,

Department of Port Logistics System,

Tongmyong University, Busan, 48520, South Korea.

Email: kjy6858@tu.ac.kr

\section{INTRODUCTION}

One of the words getting related many times to the keyword 'The fourth Industrial revolution' recently is Internet of things(IoT) [1-2]. With the existing Internets being connected to objects, a new paradigm called IoT has been newly appeared [3-4]. Thanks to the widened range of Internet, people all over the world are connected to one another. Using IoT, communication between objects is possible as well as between humans [5]. IoT provides service on every applications, using collected, processed data [6]. Objects are processor, sensor, actuator and communication function embedded devices that are connected and are exchanging data for their objective to provide useful service to human through the internet [7-8]. The recent advance of technologies is contributing to growth of field of IoT. By field of IoT growing quickly, there are some issues of sensing, connection, electricity, cost, etc [9]. IoT has infinite potential to provide service of better quality to present and future generations. Open source started with software and expanded to hardware such as Arduino and Raspberry Pi, the main players of the Internet of Things. Open source hardware is widely used in education and can quickly develop prototypes for product development [10].

This thesis is in regard to designing, materializing real-time object control system using MQTT(Message Queueing Telemetry Transport) which meets the requirements for connectivity, scalability and resource sharing by interconnecting objects, and Arduino, Raspberry Pi the representatives of OSHW(Open Source Hardware) for each sensor-actuator node and a main control node. Sensor node measures temperature, humidity and $\mathrm{CO} 2$ concentration around and transmits the measurements to the main control node through MQTT protocol. Main control node publishes data of sensor node to clouds through MQTT broker and Node-RED server. Depending on collected measurements, Node-RED can also control actuator node. In other words, object control system is not a single device but an assembly of interdependently operating devices which is cheap to construct service and easy, quick, and stable to control object. 


\section{REVIEW LITERATURE}

This section describes IoT, open source hardware, cloud platforms, MQTT and examples of open source platform.

\subsection{Internet of things}

A term IoT is the abbreviation of Internet of things coined by Professor Kebin Ashton of Massachusetts Institute of Technology(MIT), when he quoted, "A system of interrelated computing devices, mechanical and digital machines, objects, animals or people that are provided with sensors and the ability to transfer data over a network without requiring human-to-human or human-to-computer interaction" [11]. Now, its concept is being defined differently by each institutions. The core components of IoT are human, objects and service. The center of them is human. It is Internet's objective to make human's life convenient and valuable. The objects mean Internet-connected terminal devices or various types of devices that humans use. objects are meant to provide various information and service is an intelligent process through them that human needs. Objects produce various informations and activate itself using that informations for the purpose of enhancing the value of humans' life [12]. In IoT generation, a massive amount of sensor data must be produced all at once in high speed and be checked, managed and analyzed effectively in real time [13].

\subsection{Open source hardware}

The appearance of IoT brought a change of IT paradigm in Hardware, Sofeware and Network perspective. Object is a kind of a compact embedded system equipped with micro controller or micro processor. The open source concept which is getting attention in software industry is also becoming an issue in the field of hardware because of the expansion of IoT market. Being used as a tool of creating startup ideas, open source hardware is being estimated that it is contributing to IoT related startup visualization. Open source hardware is a board that includes a computing function which can control relevant device or carry out a particular function. The design is open to the public to make anybody use it freely and to enable revision and redistribution. The most famous platforms in open source hardware are 'Arduino' and 'Raspberry Pi'[14]. The Arduino which uses a MCU chip of 2005 ATMEL is a board made to enable easy connection between various sensors and different kinds of peripheral devices. For Raspberry Pi, Application Processor(AP) of Broadcom is being used, and Internet connection is possible. One of its characteristics is that beginners can also use it easily. Until now, Arduino and Raspberry Pi have been mainly used on children's training computers. But recently the trend seems that they are being adopted in the development of IoT related products [15].

\subsection{Cloud platform}

Because every object is connected to Internet, an infra for exchange of information and mutual communication among humans and objects, and among objects and objects are needed [16]. Today, users of cloud service which enables them to use functions of computer hardware and software through the Internet without constructing or maintaining server on the Internet for the various services are increasing. The cloud which can be provided anywhere that Internet connection is possible is composed of the solution for data farm and data processing. Through cloud service, ordinary persons can approach to their information, and developers are provided with broad range of possibility of developing application service for free [17].

\subsection{Node-red}

Node-RED is a representative middleware tool in IoT field for front-end development developed by IBM. Because the Node-RED is a flow-based visualization software and many devices and protocols are abstracted with the node that have in-output, it is easy for users to construct the new service or data processing flow by using web to connect these nodes and set required main parameters [18]. Node-RED is developed based on Node.js so that it can use many libraries provided by Node.js and add a new node. If Node-RED is installed, IP address can be accessed through web browser and various tools for GUI can be utilizing dashboard function so the users can construct their screen with formerly designed control flows to monitor for dash board [19].

\subsection{MQTT}

MQTT(Message Queue Telemetry Transport) is a lightweight messaging protocol optimal to telemetry devices, sensors and mobile devices. MQTT, a protocol which is getting attention as an optimal protocol in IoT environment can effectively materialize message communication environment in low-power, restricted communication environment [20]. MQTT is a Publish/Subscribe mode protocol made to be used in M2M(machine-to-machine) and IoT(Internet of Things). It is designed to be used in low-power, low-bandwidth environment during two-way pub/sub messaging service implementation for control of small devices and effective communication of sensor information, while development between heterogeneous platforms is simple [21]. MQTT's advantage is that it can be operated in low-power, on network that is not 
trusted, or in non-TCP/IP base. To use MQTT, Broker is needed like an Internet web server. Broker stands between publisher and subscriber and do a role of bridge by mediating messages [22]. There are various functions and types of brokers also like web server.

\subsection{Open source platforms}

In [23], a cloud-based people counter for the sake of targeting and marketing was proposed. It designed cloud-based people counter using Raspberry Pi imbedded system, and transmitted the received data to an IoT platform "ThingSpeak". There is a study to provide a manual to monitor, analyze and apply the sensing-informations that are collected by using various sensors, and finally realize the smart agriculture [24]. It is designed to connect four kinds of sensors to Raspberry $\mathrm{Pi}$, and visualize the informations that are collected in cloud through MQTT. [25] is one of the cases of application of IoT in smart-home, which is designed to control various home appliances using Raspberry Pi, MQTT, REST etc.

\section{REAL-TIME OBJECT CONTROL SYSTEM IMPLEMENTATION}

This study is for the low-cost system which is able to get acceptable sensor value by using open source hardware and respond in real-time by using MQTT protocol. The Figure 1 explains the composition of entire system designed in this thesis. The entire system is based on multiple open source hardware and software for maintaining short system establishment time and low price. One of the objectives of system designing is to replace PC which is traditional in system integration. Arduino and Rapsberry Pi have been chosen as open source hardware. These systems are cheap, easy to use and maintain [26-27].

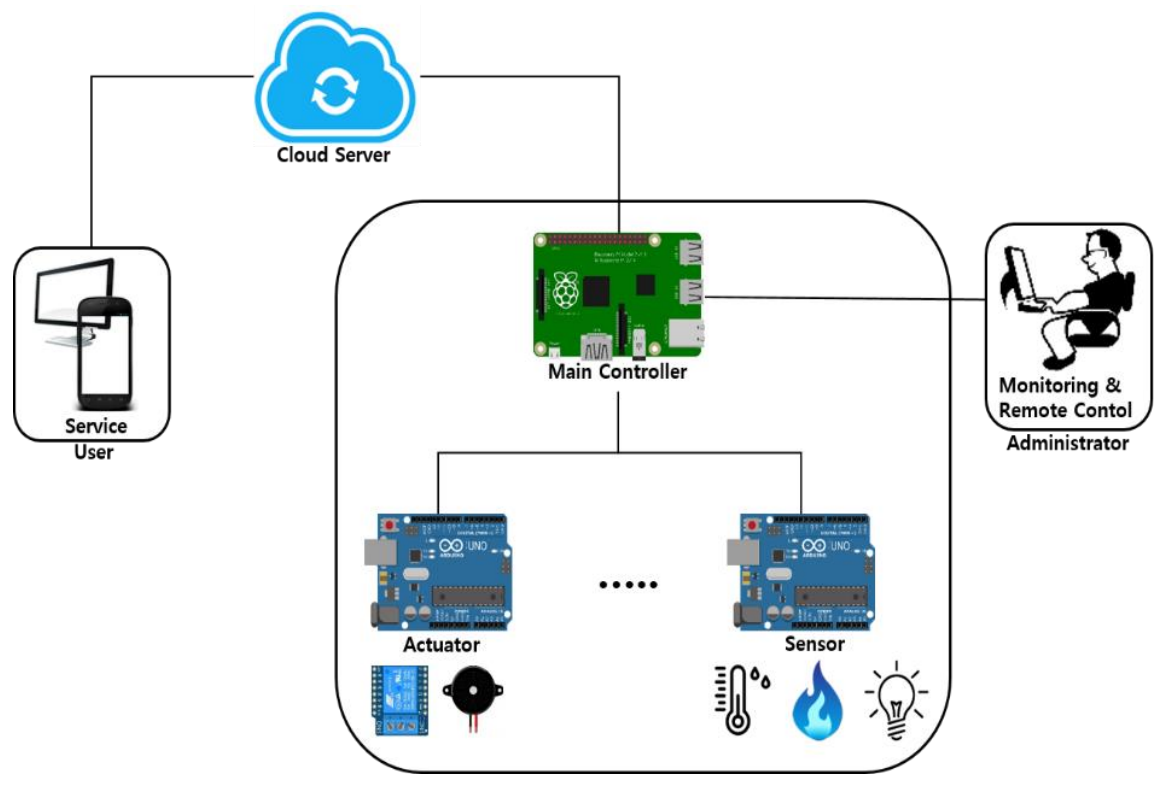

Figure 1. System configuration diagram

Also, for implementation of real time two-way communication between thing and human without being intervened by time and place, not a single-way communication, web interface, intermediate server and hardware for reception of the order from the user.

The Table 1 explains the components and main functions.

Table 1. System component

\begin{tabular}{cl}
\hline Component & \multicolumn{1}{c}{ Main function } \\
\hline User/Administrator & Gets provided the informations, can control hardware. \\
Cloud Server & Two-way communication with main controller in real time, provides user \\
& interface. \\
Bain Controller & Broker role between connected objects that provides message and protocol. \\
& Connection of database and Cloud service through Node-RED server \\
& performance. \\
Actuator/Sensor & Collects and process sensor informations and control actuator. \\
\hline
\end{tabular}

Real-time object control system using open source platform (Chang-Gyu Cgseong) 
General users are provided with the various types of sensing data collected by temperature humidity sensor, gas sensor, etc. of the thing. Administrator is the main agent who gets provided with the information of the object through the main controller and then controls and manipulates the order for buzzer or motor. Cloud server enables the users to get and check out the information of the thing collected from the main controller in real time. It provides user interface to user's mobile device or desktop in response and provides that response to the user while communicating with Broker in real time.

Main controller used Rapsberry Pi of Microprocess based platform for effective management and process of entire system, and Node which stands for objects are combination of Arduino of Micro-controller based platform and wireless module. As wireless sensor network is a smart and effective way to collect surrounding data, Arduino and Rapsberry Pi are connected by wireless communication using MQTT protocol. To use MQTT, Broker is needed like Internet web server. There are various functions and types of brokers. For the design and implementation of system in this thesis, a mosquito broker has been used. Sensor and actuator got the actuators which collect-process the data from various sensors of itself, provide informations to user and administrator and control actuators of itself following the administrator's order or programmed order.

\section{REAL-TIME OBJECT CONTROL SYSTME IMPLEMENTATION}

\subsection{System implementation}

As shown in the Figure 2 and Figure 3, the entire system has been constructed to verify the concept of open source platform. It consists of the sensor node and actuator node which are able to bo equipped on each objects, and designed to be able to send checked and processed sensors' readings to in outside through main controller using WiFi based MQTT. A cheap ESP-01 module has been used for MQTT WiFi communication between the devices. Raspberry Pi has been used as the main controller which is in charge of data collection and MQTT broker function, and Arduino has been used as sensor node and actuator node.

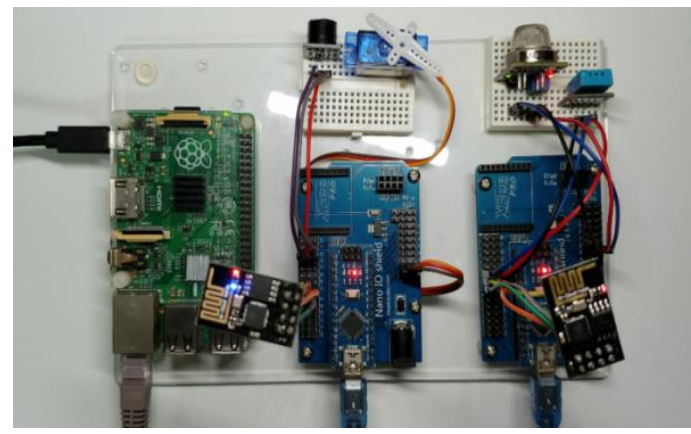

Figure 2. Full system implementation

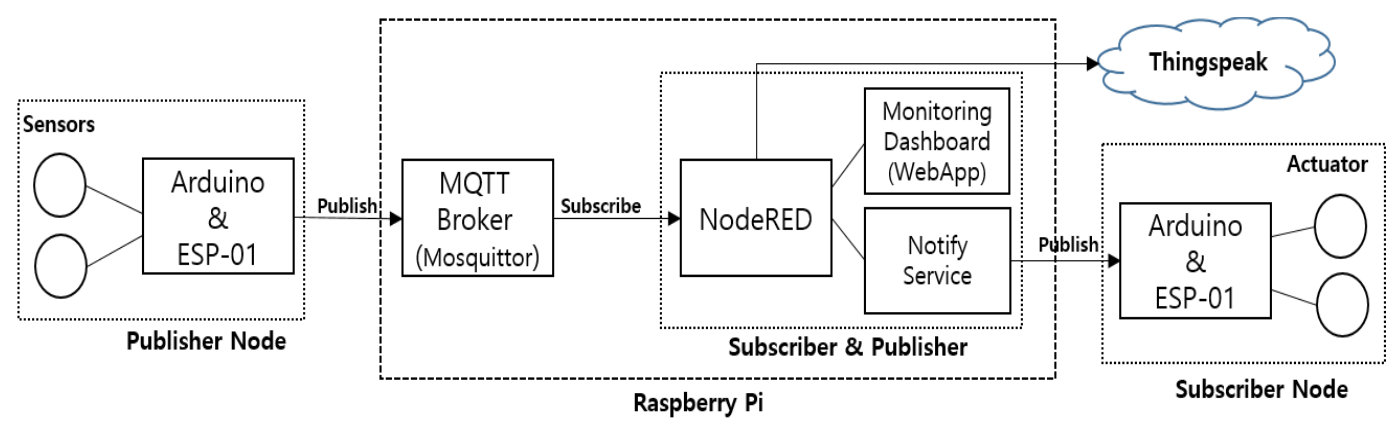

Figure 3. Detailed system design

\subsection{Object node}

Sensor node, which applies to object node collects temperature-humidity and gas informations and send it to Raspberry Pi through WiFi based MQTT. Then, Raspberry Pi sends actuation order to MQTT through actuator node for actuation of alarm and motor. Through object management using extracted 
information by carrying out caution and auto lock function in real time, the quality of service is guaranteed. When battery power supply is in use, sensor node must be used in sleep mode for efficient power management. To minimize the use of power, the range of normal and abnormal should be set so that it can stand by in sleep mode in normal range, wake itself up in abnormal range to transmit information to the main controller through MQTT, and turn itself back to sleep mode if it is in normal range and not in designated transmit period. Figure 4 is a dashboard showing the monitoring results of the sensor nodes.

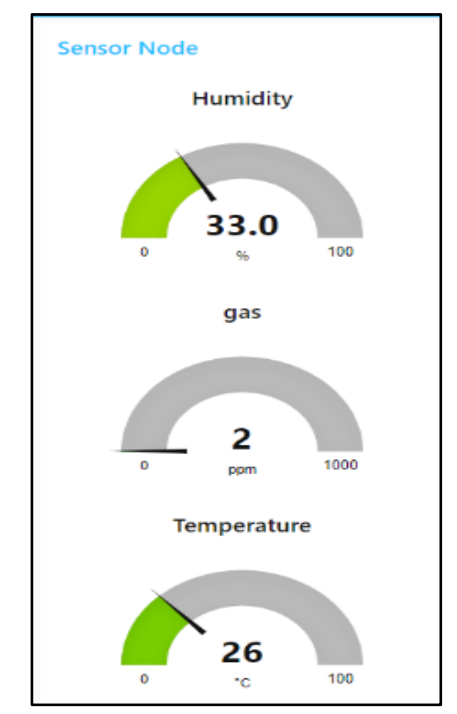

Figure 4. Node-red dashboard under monitoring

\subsection{Main controller}

Raspberry Pi has been used as the main controller. Mosquitto broker has been used for sharing of information about object resources even in a situation that direct connection between the objects is impossible. Objects exchange each other's resource informations through mosquitto broker. Node-RED server program for object node has been installed and used. Also, MySQL database that saves collected sensor informations has been constructed and interlocked with Node-RED. The main roles of this device are MQTT broker which is wirelessly connected with object node, and Node-RED server for object node, cloud and database. Cloud uses Thingspeak service platform. Thingspeak is free to use and enables access to informations of certain place's object in real time connection through the Internet. Figure 5 shows the Node-RED flow program.

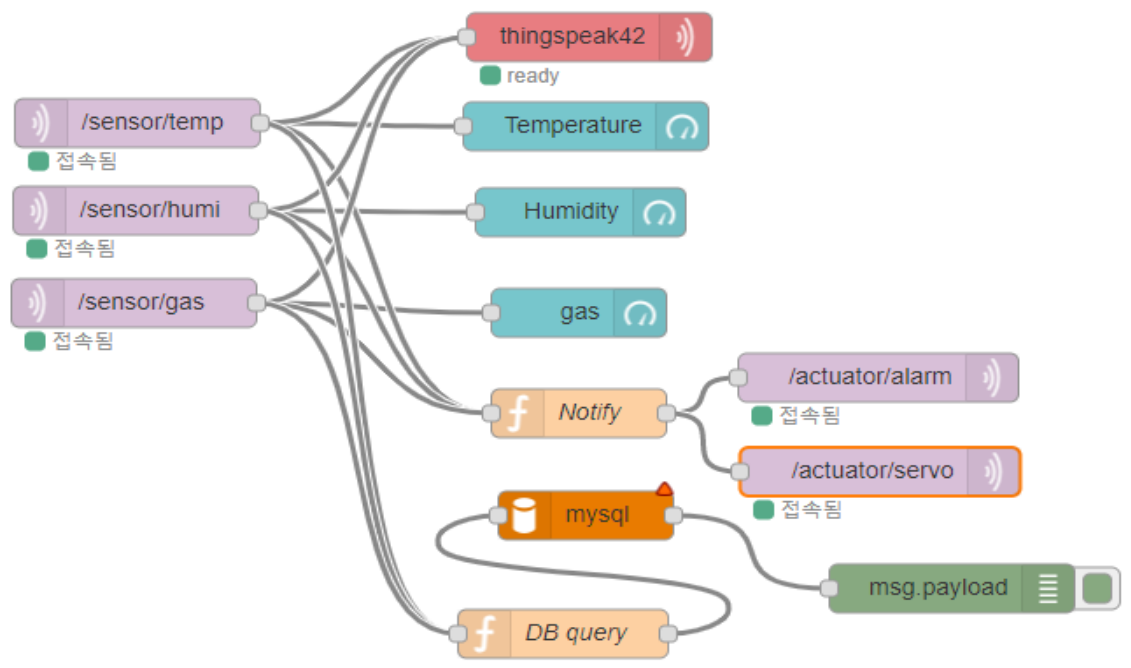

Figure 5. Node-RED flow program 


\section{CONCLUSION AND FUTURE RESEARCH}

In IoT environment, lifeless machines are connected to the Internet to save data on the web or cloud and provide useful service to human through analysis-process based on collected data. In other words, these machines are enriching human life by processing collected data to information, information to knowledge, and knowledge to wisdom.

The designed-implemented system in this thesis suggested a real time object control plan which is free from time and place limitation, by using open source. By combining existing technologies and recently standardized new technologies, the system was designed, implemented to enable the communication between different protocols and to strengthen the advantages of each protocols. Plus, by investigating the prospect and current market's current status quo of IoT industry and studying recent technologies which are applicalble in IoT environment, the implementation of the system which meets the study objective was possible.

Tried to collect informations from sensor device, control actuator by constructing MQTT broker server and implemented real time communication between broker server and Node-RED using MQTT protocol. A system suggested in this thesis would make technology development easier than before by implementing four components of cost-efficient open source, connectivity of two-way communication between object and user(or object), resource sharing through cloud and real time possibility through the web. In research from now on, service for object resource search and registration, and security function for information access control would be implemented by expanding MQTT broker structure.

\section{REFERENCES}

[1] Xu. Min, Jeanne M. David and Suk Hi Kim, "The Fourth Industrial Revolution: Opportunities and Challenges," International Journal of Financial Research, vol. 9, no. 2, pp. 90-95, 2018.

[2] MinHwa Lee, et al., "How to respond to the fourth industrial revolution, or the second information technology revolution? Dynamic new combinations between technology, market, and society through open innovation., "Journal of Open Innovation: Technology, Market, and Complexity, vol. 4, no. 21, pp. 1-24, Jun. 2018.

[3] L. W. Santoso, et al., "Smart Home System Using Internet of Things," Journal of information and communication convergence engineering, vol. 16, no. 1, pp. 60-65, Mar. 2018.

[4] Rajkumar Buyya, Satish Narayana Srirama, "Internet of Things (IoT) and New Computing Paradigms," Fog and Edge Computing: Principles and Paradigms, Wiley, pp. 1-23, 2019.

[5] T. Thaker, "Esp8266 based Implementation of Wireless Sensor Network with Linux based Web-server, " Symposium on Colossal Data Analysis and Networking, March 2016.

[6] Dutta Pramanik. Pijush, Choudhury. Prasenjit, "IoT Data Processing: The Different Archetypes and their Security \& Privacy Assessments," Internet of Things (IoT) Security: Fundamentals, Techniques and Applications, pp. 37-54, Aug. 2018.

[7] Ovidiu Vermesan, Peter Friess, "Internet of Things Applications-From Research and Innovation to Market Deployment," River Publishers, pp. 243-286, Jun. 2014.

[8] Y. S. Lee, B. Jo and S. Han, "A Light-Weight Rapid Control Prototyping System Based on Open Source Hardware," in IEEE Access, vol. 5, pp. 11118-11130, 2017.

[9] Verma Shikhar, et al., "A survey on network methodologies for real-time analytics of massive IoT data and open research issues," IEEE Communications Surveys \& Tutorials vol. 19, no. 3, pp 1457-1477, 2017.

[10] Dragan Mlakić, Srete Nikolovski, Emir Alibašić, "Designing Automatic Meter Reading System Using Open Source Hardware and Software," International Journal of Electrical and Computer Engineering, vol. 7, no. 6, pp. 3282-3291, Dec. 2017.

[11] Sundmaeker, Harald, et al., "Vision and challenges for realising the Internet of Things," Cluster of European Research Projects on the Internet of Things, European Commision, vol. 3, no. 3, pp 34-36, 2010.

[12] ATZORI, Luigi, et al., "The social internet of things (siot)-when social networks meet the internet of things: Concept, architecture and network characterization," Computer networks, vol. 56, no.16, pp. 3594-3608, 2012.

[13] Perera, Charith, et al., "Context aware computing for the internet of things: A survey," IEEE communications surveys \& tutorials vol. 16, no.1, pp. 414-454, 2013.

[14] Deshmukh, Akshay D., and Ulhas B. Shinde. "A low cost environment monitoring system using raspberry Pi and arduino with Zigbee," 2016 International Conference on Inventive Computation Technologies (ICICT), IEEE, vol. 3, 2016.

[15] MÄENPÄÄ, Hanna, et al., "Assessing IoT projects in university education: A framework for problem-based learning, " In: Proceedings of the 39th International Conference on Software Engineering: Software Engineering and Education Track, IEEE Press, pp. 37-46, 2017.

[16] BELLO. Oladayo and ZEADALLY. Sherali, "Intelligent device-to-device communication in the internet of things," IEEE Systems Journal, vol. 10, no. 3, pp. 1172-1182, 2014.

[17] Ray. Partha Pratim, "A survey of IoT cloud platforms," Future Computing and Informatics Journal, vol. 1, no. 1-2, pp. 35-46, 2016.

[18] Ray. Partha Pratim, "A survey on visual programming languages in internet of things," Scientific Programming, vol. 2017.

[19] NGU. Anne H., et al., "IoT middleware: A survey on issues and enabling technologies," IEEE Internet of Things Journal, vol. 4, no. 1, pp. 1-20, 2016. 
[20] Soni, Dipa, and Ashwin Makwana. "A survey on mqtt: a protocol of internet of things (iot)," International Conference on Telecommunication, Power Analysis and Computing Techniques (ICTPACT-2017), 2017.

[21] Salman. Tara, "Internet of things protocols and standards," Networking Protocols and Standards for Internet of Things, 2015.

[22] AL-FUQAHA, Ala, et al. "Internet of things: A survey on enabling technologies, protocols, and applications," IEEE communications surveys \& tutorials, vol. 17, no. 4, pp. 2347-2376, 2015.

[23] Abd Kadir Mahamad., et al., "Cloud-based people counter," Bulletin of Electrical Engineering and Informatics, vol. 9, no. 1, pp. 284-291, 2020.

[24] Wahidur Rahman., et al., "Real-time and low-cost IoT based farming using raspberry Pi," Indonesian Journal of Electrical Engineering and Computer Science, vol. 17, no. 1, pp. 197-204, 2020.

[25] Afrizal Mayub., et al., "Implementation smart home using internet of things," TELKOMNIKA (Telecommunication, Computing, Electronics and Control), vol. 17, no. 6, pp. 3126-3136, 2029.

[26] Soriano. Angel, et al., "Low Cost Platform for Automatic Control Education Based on Open Hardware," IFAC Proceedings vol. 47, no. 3, pp. 9044-9050, 2014.

[27] Ferdoush. Sheikh, LI. Xinrong, "Wireless sensor network system design using Raspberry Pi and Arduino for environmental monitoring applications," Procedia Computer Science, vol/ 34, pp. 103-110, 2014.

\section{BIOGRAPHIES OF AUTHORS}
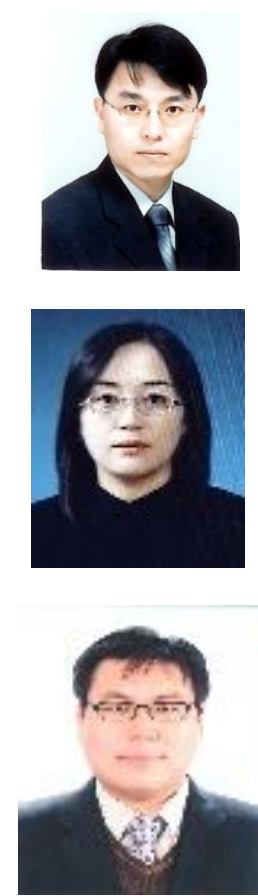

Chang-Gyu Sung received his Ph.D. in computer science from Korea Maritime University in 2017 and a MS degree in computer science from Kyungsung University in 1998. He has been a professor at Pusan National University since 2005. His research interests include the Internet of Things, computer thinking, and artificial intelligence.

Jung-Yee Kim received her bachelor's degree in the Department of Computer Science and Statistics from the KyungSung University in 1990. She received her MS degree in the Department of Computer Science and Statistics from KyungSung University in 1994. She is studying for her master's degree at Pusan National University. From 2001 to 2006, she was a professor in the Department of Computer Information Processing at TongMyong College. She has been a professor in the Department of Port Logistics System at TongMyong University since 2006. Her current research interests include IoT, RTLS, intelligent Database and phone App.

Doo-Jin Park received his Ph.D degree in the Department of Logistics System from the National Korea Maritime and Ocean University in 2007. He received his MS degree in the Department of Information Communication Engineering from Pukyong National University in 2002. He is a professor in the Department of Port Logistics System at TongMyong University since 2011. His current research interests include IT Logistics, IoT, RTLS, Ah-hoc Network, Smart Phone App. 